\title{
Traqueostomía percutánea con asistencia fibrobroncoscópica: Evaluación prospectiva de 100 casos consecutivos y revisión de la literatura
}

\author{
Carlos Romero $\mathbf{P}^{1}$, Rodrigo Cornejo $\mathbf{R}^{\mathbf{1}}$, Mauricio Ruiz $\mathrm{C}^{\mathbf{2}}$, \\ Ricardo Gálvez $A^{1}, 0$ svaldo Llanos $V^{1}$ Eduardo Tobar $A^{1}$, \\ Jorge Larrondo $\mathbf{G}^{\text {a, }}$ José Castro $0^{1}$. \\ Fiberoptic bronchoscopy assisted \\ percutaneous tracheostomy. \\ Report of 100 patients
}

Background: Development of percutaneous techniques for tracheostomy have facilitated its implementation in the intensive care unit (ICU). Aim: To evaluate the safety of performing percutaneous tracheostomy (PT) using the Ciaglia Blue Rhino thechnique with fiberoptic bronchoscopy assistance in patients with prolonged mechanical ventilation. Patients and methods: Prospective evaluation of 100 consecutive patients aged $62 \pm 16$ years (38 women) subjected to percutaneous tracheostomy. All the procedures were performed in the ICU. Demographic variables, APACHE II, days of mechanical ventilation before PT, operative and post operative complications were recorded. Results: Mean APACHE II score was 20 \pm 3 . Patients required on average $16 \pm 7$ days of mechanical ventilation before PT. Eight patients (8\%) had operative complications. One had an episode of transitory desaturation, one had a transitory hypotension related to sedation and six had mild bleeding not requiring transfusion. No patient required conversion to surgical tracheostomy. Four patients (4\%) presented post operative complications. Two had a mild and transitory bleeding of the ostomy, and two had a displacement of the cannula. No other complications were observed. Conclusions: PT using the Ciaglia Blue Rhino technique with fiberoptic bronchoscopy assistance is a safe procedure that can be performed in the ICU by trained intensivists (Rev Méd Chile 2008; 136: 1113-20).

(Key words: Bronchoscopy; Intensive care units; Tracheostomy, percutaneous)

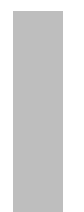

Recibido el 6 de noviembre, 2007. Aceptado el 21 de abril, 2008.

Los autores declaran no tener conflicto de intereses. Este estudio no fue financiado por una compañía médica.

${ }^{1}$ Unidad de Pacientes Críticos. ${ }^{2}$ Sección Enfermedades Respiratorias, Departamento de Medicina, Hospital Clínico Universidad de Chile. Santiago, Chile.

aEstudiante de Medicina, Facultad de Medicina, Universidad de Chile.

Correspondencia a: Dr. Carlos Romero. Unidad de Pacientes Críticos, Hospital Clínico Universidad de Chile, Facultad de Medicina, Universidad de Chile. Santos Dumont 999, Independencia. Fono/Fax: 562- 9788264. Santiago Norte, Chile. E mail: caromero@redclinicauchile.cl 
L a traqueostomía es uno de los procedimientos quirúrgicos más comúnmente practicados en pacientes críticos $^{1}$. En diferentes series a nivel nacional e internacional, la prevalencia reportada oscila entre $10 \%$ y $20 \% 2-4$, aunque puede ser mayor en algunos subgrupos de enfermos como aquellos con trauma $(24 \% \text { a } 48 \%)^{5-7}$.

Gracias al avance de la medicina intensiva, actualmente es posible brindar a nuestros enfermos soporte vital por largos períodos de tiempo. En este escenario una proporción considerable de pacientes críticos pueden requerir intubación translaríngea y ventilación mecánica (VM) prolongada. Varios estudios ${ }^{5,8-12}$ han reportado que la realización temprana de una traqueostomía en pacientes seleccionados puede acortar el tiempo de VM y reducir la estadía en la Unidad de Cuidados Intensivos (UCI). Incluso algunos investigadores han encontrado una menor incidencia de neumonía asociada a VM y una reducción de la mortalidad $^{4,8,12-16}$. Un reciente metaanálisis reportó una reducción de los días de VM y estadía en la UCI con la realización de una traqueostomía temprana, sin embargo no pudo demostrar una menor incidencia de neumonía asociada a VM ni reducción de la mortalidad ${ }^{17}$.

El desarrollo de diversas técnicas de traqueostomía percutánea (TPC), ha facilitado la realización del procedimiento en pacientes críticos, acortando los plazos para su ejecución, favoreciendo la utilización de los recursos y evitando movilizar a los pacientes fuera de la UCI ${ }^{1,18}$.

El objetivo del presente estudio, fue evaluar la seguridad de la TPC, mediante la técnica de Ciaglia modificada con asistencia fibrobroncoscópica, en pacientes críticos sometidos a VM prolongada.

\section{PACIENTES Y MÉTOdO}

Entre octubre de 2004 y febrero de 2007, se evaluaron e incluyeron en forma prospectiva todos los pacientes mayores de edad sometidos a $\mathrm{VM}$, en quienes fue necesaria la realización de una traqueostomía. Se excluyeron los pacientes con contraindicación absoluta para una TPC. La indicación clínica de la TPC se basó en los siguientes criterios: 1) VM prolongada (VM $>2$ semanas), 2) Fracaso del destete de la VM con reintubación en dos oportunidades, 3) Compromiso cuantitativo de conciencia con incapacidad para proteger la vía aérea, y 4) Patología neuromuscular en la que se anticipó la necesidad de VM prolongada. Contraindicaciones absolutas para la realización de una TPC fueron: edad menor a 18 años, distorsión de la anatomía cervical por masas o cirugía, infección cutánea cervical y traqueostomía previa.

Todas las TPC fueron electivas y se realizaron en la UCI de manera estandarizada con la técnica de Ciaglia modificada (dilatación única) empleando el equipo Blue Rhino (Cook Critical Care, Bloomington, IN, USA). En todos los casos se obtuvo consentimiento informado de los familiares directos.

Las TPC fueron practicadas por dos intensivistas formados y entrenados previamente en el procedimiento (C.R. y R.C.). En todos los casos se contó con la presencia de un especialista en enfermedades respiratorias para la asistencia fibrobroncoscópica (Broncoscopio 1T30, Olympus Medical Systems Corp, Tokio, Japón). El método empleado para la realización de la TPC ha sido descrito extensamente en la literatura ${ }^{19}$.

Se registraron variables demográficas, APACHE II y los días en VM hasta la realización de la traqueostomía. En todos los casos los días de intubación fueron equivalentes a los días VM. Los pacientes fueron seguidos hasta su decanulación, traslado a otro hospital o fallecimiento.

La complicaciones operatorias y postoperatorias evaluadas fueron definidas como sigue: 1) Pérdida de la vía aérea: extubación accidental durante el procedimiento con incapacidad para acceder a la tráquea por más de 30 s. 2) Conversión a técnica quirúrgica: necesidad de suspender la TPC y realizar un abordaje quirúrgico en la UCI o en pabellón. 3) Hemorragia mayor: sangrado que por sí mismo ocasione hipotensión, requiera la transfusión de $\geq 2$ unidades de glóbulos rojos o requiera conversión quirúrgica para su control. 4) Hemorragia menor: sangrado que se detenga con compresión o alguna maniobra hemostática, sin causar inestabilidad hemodinámica, requerir transfusión de glóbulos rojos ni cirugía. 5) Neumotórax/neumomediastino: presencia de aire en la cavidad pleural o mediastínica asociada directamente al procedimiento. 6) Hemotórax: evidencia de sangre en el espacio pleural ocasio- 
nada directamente por el procedimiento. 7) Hipotensión: caída de la presión arterial media bajo 70 mmHg en algún momento del procedimiento. 8) Hipoxemia: caída de saturación de oxígeno bajo $90 \%$ en algún momento del procedimiento. 9) Desplazamiento de la cánula: excursión accidental de la cánula de traqueostomía. 10) Infección de la herida: cambios inflamatorios locales, secreción purulenta por la herida con incremento de parámetros inflamatorios o documentación microbiológica en muestra tomada del estoma de la traqueostomía. 11) Muerte: fallecimiento del paciente por una complicación directamente relacionada a la TPC.

Un tercio de los pacientes de la serie (33 casos) tuvieron alguna contraindicación relativa clásica para la realización de una TPC, por lo que se consideraron "pacientes de alto riesgo" (Tabla 1). Diecinueve de los casos correspondieron a pacientes con obesidad (índice de masa corporal $>30$ ). Siete pacientes se encontraban cursando con una coagulopatía de consumo (INR $>2$ o plaquetas $<50.000$ ), al momento en que se decidió realizar la TPC. En todos los casos se alcanzó un INR $<1,5$ y un recuento de plaquetas $>50.000$ mediante transfusión de plasma fresco congelado y plaquetas antes del procedimiento. Cuatro pacientes tuvieron cuello corto (distancia entre el cartílago cricoides y el ángulo esternal menor a 2,5 cm), y en otros tres pacientes no fue posible realizar hiperextensión del cuello, en un caso por una lesión cervical traumática y en los otros dos por espondiloartrosis.
Estadística. Los datos son presentados como frecuencias y porcentajes para las variables categóricas, y promedio y desviación estándar (DE) para las variables continuas. Se empleó t de Student bilateral para la comparación de las variables continuas y test exacto de Fisher para el análisis de las variables categóricas. Los cálculos estadísticos fueron realizados usando SPSS 14.0 (Chicago, Ill., USA) para Windows XP. Un valor $\mathrm{p}<0,05$ fue considerado estadísticamente significativo.

\section{RESULTADOS}

Durante el período de estudio 100 pacientes se sometieron a una TPC con asistencia fibrobroncoscópica en el Hospital Clínico de la Universidad de Chile.

La edad del grupo fue $62 \pm 16$ años, 38 fueron mujeres y 62 varones. El puntaje APACHE II fue $20 \pm 3$. En 67 pacientes el motivo de la VM fue insuficiencia respiratoria por síndrome de distrés respiratorio agudo asociado a sepsis. En el resto de los pacientes la causa de VM fue patología aguda del sistema nervioso central o periférico. Los pacientes permanecieron en promedio $16 \pm 7$ días en VM antes de la realización de la traqueostomía (Tabla 1).

Complicaciones operatorias. Ocho pacientes (8\%) presentaron alguna complicación durante el procedimiento. Un enfermo experimentó un episodio de desaturación transitoria que mejoró al retirar el fibrobroncoscopio del tubo orotraqueal y reanudar la

Tabla 1. Características demográficas y complicaciones por subgrupos

\begin{tabular}{|lccc|}
\hline Variables & Bajo riesgo $(\mathrm{n}=67)$ & Alto riesgo $(\mathrm{n}=33)$ & $\mathrm{p}$ \\
\hline Mujeres & $28(42 \%)$ & $10(30 \%)$ & 0,28 \\
Edad & $62 \pm 17$ años & $63 \pm 13$ años & 0,93 \\
APACHE II & $20 \pm 5$ & $19 \pm 2$ & 0,27 \\
VM-TPC & $15 \pm 4$ & $17 \pm 6$ & 0,05 \\
Complicaciones operatorias & $9 \%(6 / 67)$ & $6 \%(2 / 33)$ & 1 \\
Complicaciones postoperatorias & $3 \%(2 / 67)$ & $6 \%(2 / 33)$ & 0,59 \\
Complicaciones globales & $12 \%(8 / 67)$ & $12 \%(4 / 33)$ & 1 \\
\hline
\end{tabular}

*Días de intubación y ventilación mecánica hasta la realización de la traqueostomía percutánea. 
VM. Un paciente desarmolló hipotensión transitoria relacionada con la sedación que revirtió rápidamente con fluidos. Seis pacientes presentaron sangrado menor. En cuatro casos el mismo cedió con la compresión de la zona, en tanto que en dos pacientes fue necesaria la aplicación de puntos hemostáticos. No se registraron complicaciones de riesgo vital.

Complicaciones postoperatorias. Cuatro pacientes (4\%) presentaron alguna complicación postoperatoria. Dos enfermos presentaron sangrado menor y transitorio del estoma de la TPC y en dos casos se produjo un desplazamiento de la cánula de traqueostomía. No se registró otro tipo de complicaciones.

Pacientes de alto riesgo. Del subgrupo de enfermos con alguna contraindicación relativa para la realización de una TPC ( $\mathrm{n}=33$ ), sólo 2 pacientes obesos (6\%) presentaron un sangrado menor, en ninguno de los otros casos se identificó una complicación operatoria. Dos (6\%) pacientes hiperobesos (índice de masa corporal $>50$ ) experimentaron el desplazamiento de la cánula de traqueostomía con desaturación transitoria asociada. No se documentó otra complicación postoperatoria en este subgrupo de enfermos (Tabla 1).

\section{DiscUSIÓN}

En este trabajo comunicamos la mayor serie nacional con evaluación sistemática de las complicaciones operatorias y postoperatorias de la TPC, mediante la técnica de Ciaglia modificada y asistencia fribrobroncoscópica, en pacientes críticos sometidos a VM prolongada. Encontramos que el procedimiento es seguro cuando es realizado por personal médico con entrenamiento adecuado y puede ser ejecutado en la gran mayoría de casos. De las 115 traqueostomías realizadas en pacientes de la UCI durante el período de estudio, 100 pudieron ser ejecutadas por vía percutánea sin incidentes mayores.

La TPC ha sido perfeccionada en forma considerable desde su introducción en los años 50'. Diferentes técnicas han sido descritas, con tasas de complicaciones variables según el método utilizado ${ }^{20-26}$.

En nuestro medio el único equipo comercializado para TPC es el Blue Rhino (Cook Critical Care, Bloomington, IN, USA), ya que su predecesor que empleaba dispositivos de diferente calibre para dilatación múltiple progresiva fue retirado del mercado. El sistema modificado de dilatación única ha mostrado tener ventajas sobre el sistema de dilatación múltiple ${ }^{27,28}$. Adicionalmente, varios estudios han encontrado que la técnica de Ciaglia modificada presenta un mejor perfil de seguridad al compararla con otras técnicas de $\mathrm{TPC}^{29-33}$. Westphal y cols, observaron que la técnica de Ciaglia modificada y la técnica de Fantoni, desarrollada en Italia, muestran un comportamiento equivalente en términos de seguridad ${ }^{34,35}$.

La incertidumbre sobre la seguridad de la TPC en relación a la TQx se ha ido despejando en los últimos años ${ }^{36-38}$. Silvester y cols ${ }^{39}$, randomizaron 200 pacientes a TPC con la técnica de Ciaglia o TQx. Todos los procedimientos fueron realizados en la UCI por intensivistas entrenados o cinujanos formados. No encontraron diferencias significativas en la tasa de complicaciones, sin embargo el grupo de TQx presentó una incidencia mayor de infección de la herida y cicatrización antiestética. El seguimiento de los pacientes a 20 meses no reveló complicaciones tardías en los grupos, y ningún enfermo mostró evidencia de estenosis traqueal. Recientemente, un metaanálisis ${ }^{40}$ de 17 estudios randomizados comparando TPC con TQx, que incluyó 1.212 enfermos, encontró que ambas técnicas eran equivalentes en términos de complicaciones hemonágicas penioperatorias, sin embargo la TPC se asoció a una reducción significativa de la tasa de infección del estoma (OR $=0,28$ [95\% IC 0,16 a 0,49; $\mathrm{p} \varangle 0,0005]$ ]. Adicionalmente, cuando se analizó el subgrupo de enfermos que fue sometido a TPC en la UCI versus el subgrupo de enfermos que fue sometido a TQx en pabellón, se encontró una reducción significativa de sangrado operatorio ( $\mathrm{OR}=0,29$ [95\% IC 0,12 a 0,$75 ; \mathrm{p}=0,01$ ) y riesgo de muerte $(\mathrm{OR}=0,71[95 \%$ IC 0,5 a 1,$0 ; \mathrm{p}$ $=0,05]$ ). Dado el cúmulo de información demostrando que la TPC es al menos tan segura como la TQx, algunos autores consideran actualmente a la TPC como la técnica de elección en pacientes críticos ${ }^{1}$.

La descripción inicial del método de TPC por Ciaglia $^{22}$, no incluía el empleo de asistencia fibrobroncoscópica para el procedimiento. Hacia finales de la década 1980-89, la incorporación de la fibrobroncoscopia para guiar el procedimiento y adicionar mayor seguridad a la TPC fue sugerida por varios autores ${ }^{41-44}$. Barba y cols ${ }^{45}$, encontraron una menor incidencia de complicaciones en el grupo de pacientes sometidos a TPC con asistencia 
fibrobroncoscópica comparado con el grupo sometido a TPC a ciegas. A nivel nacional complicaciones serias han sido reportadas con el empleo de TPC a ciegas ${ }^{46}$. Recientemente, un metaanálisis de 23 estudios con 2.237 pacientes sometidos a TPC analizó la tasa de complicaciones de la TPC con o sin asistencia fibrobroncoscópica ${ }^{47}$. En el grupo de TPC a ciegas la tasa de complicaciones fue de 16,8\% (233 en 1.385 enfermos), mientras que en el grupo de TPC con asistencia fibrobroncoscópica la tasa de complicaciones fue de 8,3\% (71 en 851 pacientes) ( $\mathrm{p}<0,0001$ ).

Kost ${ }^{47}$ evaluó una serie de 500 pacientes críticos sometidos a TPC con la técnica de Ciaglia y asistencia fibrobroncoscópica. La tasa global de complicaciones encontrada fue 9,2\%, muy similar a los resultados encontrados en nuestro estudio. En acuerdo con el estudio canadiense ${ }^{47}$, nosotros tampoco tuvimos complicaciones operatorias graves. La baja tasa global de complicaciones observada en el presente trabajo y la ausencia de complicaciones operatorias graves como neuomotórax, neumomediastino, falsa vía, enfisema subcutáneo o muerte, refuerza la importancia del empleo de la fibrobroncoscopia como un elemento adjunto a la TPC para conferirle mayor seguridad. Es probable que la TPC a ciegas con la técnica de Ciaglia modificada mantenga niveles aceptables de seguridad en manos experimentadas, como ha sido reportado por algunos auto$\mathrm{res}^{48}$, sin embargo es posible que ello requiera experiencia con la técnica quirúrgica y un mayor período de entrenamiento.

La tasa de infección de la herida reportada en el estudio de $\mathrm{Kost}^{47}$ fue de 0,8\%, esto concuerda con la ausencia de infección del estoma en nuestro estudio de 100 enfermos consecutivos.

Consideramos importante recalcar que ésta es la primera comunicación nacional, que analiza los resultados de la TPC con la técnica de Ciaglia modificada y asistencia fibrobroncoscópica, en un grupo de enfermos de alto riesgo para experimentar complicaciones perioperatorias, y que representaron un tercio de la serie total. Algunos autores han encontrado que la TPC puede ser un procedimiento seguro aun en pacientes de alto riesgo cuando es realizada por operadores calificados $^{49-51}$. En relación a este punto es importante destacar que los buenos resultados reportados en estas series $^{49-51}$, están dados porque los procedi- mientos fueron ejecutados por expertos y no por operadores en fase de entrenamiento o durante la curva de aprendizaje. Massick y $\operatorname{cols}^{52}$, observaron que inclusive en pacientes bien seleccionados (excluyendo enfermos de alto riesgo), la tasa de complicaciones es mayor en la fase inicial de implementación de la TPC. Previamente nosotros reportamos el análisis de los primeros 80 pacientes sometidos a TPC con la técnica de Ciaglia modificada y asistencia fibrobroncoscópica. Encontramos una mayor tasa de complicaciones en los primeros 25 casos (12\%) comparados con los siguientes 55 casos $(5,4 \%)$, esta diferencia fue estadísticamente significativa $(p=0,02)^{53}$.

En el presente trabajo la tasa de complicaciones globales (Tabla 1) en el subgrupo de enfermos de alto riesgo no fue diferente de la tasa de complicaciones observada en el subgrupo de enfermos de bajo riesgo, apoyando la seguridad de la TPC con la técnica de Ciaglia modificada y asistencia fibrobroncoscópica también en pacientes de alto riesgo, lo que estaría en acuerdo con lo reportado por otros estudios $^{49-51}$. Sin embargo, es muy importante acotar que ninguno de los pacientes de alto riesgo fue incluido en el presente estudio durante la curva de aprendizaje. Por tanto, nuestros resultados sobre este subgrupo de enfermos, son sólo generalizables a operadores experimentados.

En nuestra serie dos pacientes hiperobesos sufrieron el desplazamiento de la cánula de traqueostomía y desarrollaron desaturación transitoria relacionada al evento, que fue corregida con la reinstalación de la cánula. Esta complicación fue ocasionada porque debido al grosor del panículo adiposo del cuello en estos enfermos la cánula de traqueostomia estándar resultó corta, lo que favoreció su excursión. El empleo de cánulas de traqueostomía de mayor longitud (Shiley ${ }^{\circledR} \mathrm{XLT}$, Nellcor, CA, USA), probablemente previno el desarrollo de esta complicación en los otros pacientes. Actualmente empleamos cánulas XLT en forma rutinaria en todos los pacientes obesos mórbidos (Figura 1).

La TPC presenta además otras ventajas en relación a la TQx en pacientes críticos bien seleccionados. Diversos investigadores han reportado que la TPC requiere menor tiempo para su realización ${ }^{54-56}$. Aunque la diferencia de unos pocos minutos entre uno y otro procedimiento carece de relevancia clínica en el contexto de una traqueostomía electiva, puede ser de 


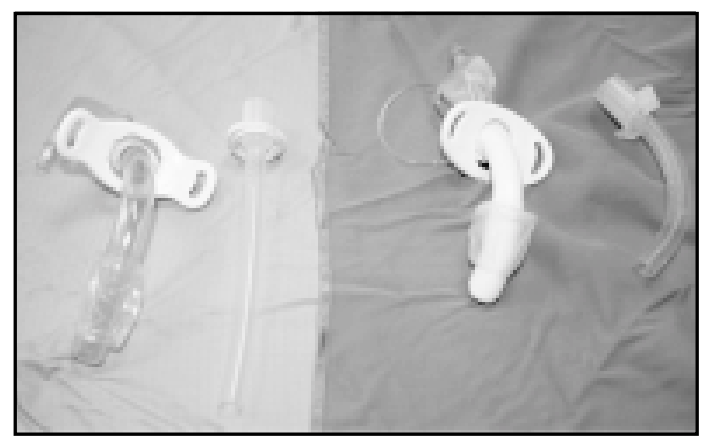

Figura 1. Comparación de una cánula convencional con endocánula (derecha) y una cánula XLT (extendedlength tracheostomy tube). La cánula convencional mide $79 \mathrm{~mm}$ de longitud versus $105 \mathrm{~mm}$ de la cánula XLT. Su mayor longitud en el plano horizontal, permite compensar el grosor del panículo adiposo del cuello en pacientes obesos mórbidos, y de esta manera evitar su desplazamiento.

gran trascendencia durante una situación de urgencia, sin embargo la TPC no es el método de primera elección para asegurar la vía aérea en este escenario. A nuestro modo de ver, lo que sí resulta interesante desde el punto de vista clínico y de gestión, es que la TQx en muchos casos puede asociarse a un mayor tiempo de espera desde que se toma la decisión de la traqueostomía hasta que finalmente es realizada en pabellón $36,54,57,58$, lo que puede retardar la salida del paciente de VM e incrementar la estadía en la UCI, con las consiguientes implicancias económicas. Previamente hemos reportado el tiempo de retardo que pueden expenimentar nuestros pacientes críticos desde la interconsulta para la TQx hasta el momento de su realización en pabellón. El grupo de TQx $(\mathrm{n}=24)$ tuvo un tiempo de espera de 5,79 $\pm 3,4$ días, mientras que en el grupo de TPC realizada en la UCI ( $\mathrm{n}=25)$, la espera fue de $1,24 \pm 0,43$ días ( $\mathrm{p} \varangle 0,0001$ ). Interesantemente esto se asoció a un mayor tiempo de VM (29 vs 22 días, $p \varangle 0,05$ ) y estadía en la UCI (30 vs 25 días, $p=0,06)$ en el grupo de TQ $x^{59}$. Algunos autores han reportado tiempos de espera hasta de una semana ${ }^{18}$. En relación al incremento de los tiempos de hospitalización en unidades de alta complejidad y costos, así como a la mejor utilización de los recursos de

\section{REFERENCIAS}

1. BARDell T, Drover JW. Recent developments in percutaneous tracheostomy: improving techniques and expanding roles. Curr Opin Crit Care 2005; 11 : 326-32. pabellón, varios estudios han mostrado un ahomo significativo con la implementación de la TPC en la $\mathrm{UCI}^{38,45,52,60}$.

Por otra parte la realización de una TPC en la UCI evita la necesidad de trasladar enfermos cníticos fuera de la unidad. Existen varios reportes alertando sobre los riesgos de transportar pacientes críticos fuera de la $\mathrm{UCI}^{61,62}$. Sin duda que siempre será necesario trasladar enfermos fuera de la UCI a procedimientos que no pueden ser realizados en la misma unidad, en estos casos los beneficios superan a los riesgos. En el caso de la traqueostomía, en tanto no existe justificación para movilizar a un enfermo fuera de su ambiente óptimo, por un procedimiento que se puede realizar en la UCI con el mismo nivel de seguridad, excepto para los pacientes con contraindicación absoluta para TPC.

En conclusión, la TPC con la técnica de Ciaglia modificada y asistencia fibrobroncoscópica está asociada a una baja tasa de complicaciones en pacientes críticos sometidos a VM prolongada. La TPC es un procedimiento seguro que puede ser realizado en la UCI por intensivistas entrenados y debería ser considerado el método de elección en este grupo de enfermos.

2. Norero E, Altschwager P, Romero C, Mellado P, Hernández G, Castilo L, Bugedo G. Ventilación mecánica en patologías agudas del sistema nervioso central: sobrevida y pronóstico funcional. Rev Méd Chil 2004; 132: 11-8.

3. Frutos-Vivar F, Esteban A, Apezteguía C, Anzueto A, Nightingale P, Gonzádez M ET al. Outcome of mecha- 
nically ventilated patients who require a tracheostomy. Crit Care Med 2005; 33: 290-8.

4. Flaatten $\mathrm{H}, \mathrm{GJ}$ erde S, Heimdal JH, Aardal S. The effect of tracheostomy on outcome in intensive care unit patients. Acta Anaesthesiol Scand 2006; 50: 92-8.

5. Arabi Y, Haddad S, Shirawi N, Shimemeri AA. Early tracheostomy in intensive care trauma patients improves resource utilization: a cohort study and literature review. Crit Care 2004; 8: R347-52.

6. Nathens AB, Rivara FP, Mack CD, Bubenfeldgd, Wang J, JuRcovich GL, MaIER RV. Variations in rates of tracheostomy in the critically ill trauma patients. Crit Care Med 2006; 34: 2919-24.

7. Shirawi N, Arabi Y. Bench-to-bedside review: early tracheostomy in critically ill trauma patients. Crit Care 2006; 10: 201-12.

8. Rodríguez JL, Steinberg SM, Luchetti FA, Gibbons KJ, TAHERI PA, FLINT LM. Early tracheostomy for primary airway management in the surgical critical care setting. Surgery 1990; 108: 655-9.

9. D'Amelio LF, Hammond JS, Spain DA, Sutyak JP. Tracheostomy and percutaneous endoscopic gastrostomy in the management of the head-injured trauma patient. Am Surg 1994; 60: 180-5.

10. Armstrong PA, Mccarthy MC, Peoples JB. Reduced use of resources by early tracheostomy in the ventilator-dependent patients with blunt trauma. Surgery 1998; 124: 763-7.

11. Bouderka MA, Fakhir B, Bouaggad A, Hmamouchi B, Hamoudi D, Harti A. Early tracheostomy versus prolonged endotracheal intubation in severe head injury. J Trauma 2004; 57: 251-4.

12. Rumbak MJ, Newton M, Truncale T, Schwartz SW, Adams JW, Hazard PB. A prospective, randomized, study comparing early percutaneous dilation tracheostomy to prolonged translaryngeal intubation (delayed tracheostomy) in critically ill medical patients. Crit Care Med 2004; 32: 1689-94.

13. Kluger Y, Paul DB, Lucke J, Cox P, Colelia JJ, TOWNSEND RN ET AL. Early tracheostomy in trauma patients. Eur J Emerg Med 1996; 3: 95-101.

14. Koldef MH, Ahrens TS, Shannon W. Clinical predictors and outcomes for patients requiring tracheostomy in the intensive care unit. Crit Care Med 1999; 27: 1714-20.

15. Boynton JH, Hawkins K, Eastridge BJ, O’Keefe GE. Tracheostomy timing and the duration of weaning in patients with acute respiratory failure. Crit Care Med 2004; 8: 261-7.

16. Chintamani, Khanna J, Singh JP, Kulshreshtha P, Kalra P, Priyambada B et al. Early tracheostomy in closed head injuries: experience at a tertiary center in a developing country. A prospective study. BMC Emerg Med 2005; 5: 1471-79.

17. Griffiths J, Barber VS, Morgan L, Young JD. Systematic review and meta-analysis of studies of the timing of tracheostomy in adult patients undergoing artificial ventilation. BMJ 2005; 330: 1243-46.

18. Al-Ansari MA, Hijazi MH. Clinical review: percutaneous diltational tracheostomy. Crit Care 2006; 10 : 202-10.

19. DuRBIN CG. Techniques for performing tracheostomy. Respir Care 2005; 50: 488-96.

20. Sheldon C, Pudenz R, Freshwater D. A new method for tracheostomy. J Neurosurg 1955; 12: 428-31.

21. Toye FJ, Weinstein JD. A percutaneous tracheostomy device. Surgery 1969; 65: 384-9.

22. Ciaglia P, Firsching R, Sinyec C. Elective percutaneous dilatational tracheostomy: A new simple bedside procedure; preliminary report. Chest 1985; 87: 715-9.

23. Schachner A, Ovil Y, Sidi J, Rogev M, Heilbronn Y, LevY MJ. Percutaneous tracheostomy: a new method. Crit Care Med 1989; 17: 1052-6.

24. Griggs WM, Worthley L, Giligan JE, Thomas PD, Myburg JA. A simple percutaneous tracheostomy technique. Surg Gynecol Obstet 1990; 170: 543-5.

25. Fantoni A, Ripamonti D. A non-derivative, non surgical tracheostomy: the translaryngeal method. Intensive Care Med 1997; 23: 386-92.

26. Frova G, Quintel M. A new simple method for percutaneous tracheostomy: controlled rotating dilation. A preliminary report. Intensive Care Med 2002; 28: 299-303.

27. Byhahn C, Wilke H J, Halbig S, Lischke V, Westphal K. Percutaneous tracheostomy: Ciaglia Blue Rhino versus the basic Ciaglia technique of percutaneous dlational tracheostomy. Anesth Analg 2000; 91: 882-6.

28. Johnson JL, Cheatham ML, Sagraves SG, Block EF, NELSON LD. Percutaneous dilational tracheostomy: a comparison single-versus multiple-dilator techniques. Crit Care Med 2001; 29: 1251-4.

29. Leinhardt DJ, Mughal M, Bowles B, Glew R, Kishen R, Macbeath J, Irvingm. Appraisal of percutaneous tracheostomy. Br J Surg 1992; 79: 255-8.

30. HutChinson RC. Life-threatening complications from percutaneous tracheostomy. Crit Care Med 1991; 19: 1220.

31. Kost KM. Percutaneous tracheostomy: comparison of Ciaglia and Griggs techniques. Crit Care 2000; 4: 143-6.

32. Nates JL, Cooper J, Myles PS, Scheinkestel CD, Tuxen DV. Percutaneous tracheostomy in critically ill patients: a prospective randomized comparison of two techniques. Crit Care Med 2000; 28: 3734-9.

33. Byhahn C, Westphal K, Meininger D, Gürke B, Kessier P, LISCHKE V. Single-dilator percutaneous tracheostomy: a comparison PercuTwist and Ciaglia Blue Rhino techniques. Intensive Care Med 2002; 28: 1262-6.

34. Westrhal K, Byhahn C, Wiшke HJ, Lischke V. Percutaneous tracheostomy: a clinical comparison of dilatational (Ciaglia) and translaryngeal (Fantoni) techniques. Anesth Analg 1999; 89: 938-43. 
35. Westphal K, Byhahn C, Rinne T, Wilke HJ, WimmerGREINECKER G, LISCHKe V. Tracheostomy in cardiosurgical patients: surgical tracheostomy versus Ciaglia and Fantoni methods. Ann Thorac Surg 1999; 68: 486-92.

36. Freeman BD, Isabelia K, Lin Natatia, Buchman TG. A meta-analysis of prospective trials comparing percutaneous and surgical tracheostomy in critically ill patients. Chest 2000; 118: 1412-8.

37. Antonelu M, Michetti V, Di Palma A, Conti G, Pennis MA, ArCangeli A et al. Percutaneous translaryngeal versus surgical tracheostomy: a randomized trial with 1-yr double-blind follow-up. Crit Care Med 2005; 33: 1015-20.

38. Higgins KM, Punthakee $\mathrm{X}$. Meta-analysis comparison of open versus percutaneous tracheostomy. Laryngoscope 2007; 117: 447-54.

39. Silvester W, Goldsmith D, Uchino S, Beilomo R, Knight S, Seevanayagam S ET aL. Percutaneous versus surgical tracheostomy: a randomized controlled study with longterm follow-up. Crit Care Med 2006; 34: 2145-52.

40. Delaney A, Bagshaw S M, Nalos M. Percutaneous dilatational tracheostomy versus surgical tracheostomy in critically ill patients: a systematic review and meta-analysis. Crit Care 2006; 10: R55-R67.

41. Paul A, Marelu D, Chiu RC, Vestweber KH, Mulder DS. Percutaneous endocopic tracheostomy. Ann Thorac Surg 1989; 47: 314-5.

42. Winkler WB, Karnik R, Seelmann O, Havlicek J, Slany J. Bedside percutaneous dilatational tracheostomy with endoscopic guidance: experience with 71 ICU patients. Intensive Care Med 1994; 20: 476-9.

43. Bouvette M, Fuhrman TM. Preventing complications during percutaneous tracheostomy. Anesthesiology 1999; 90: 918-9.

44. Kost KM. The optimal technique of percutaneous tracheostomy. Int J Intens Care 2001; 8: 82-8.

45. Barba CA, Angood PB, Kauder DR, Latenser B, Martin $\mathrm{K}$, MCgonigal MD ET AL. Bronchoscopic guidance makes percutaneous tracheostomy a safe, costeffective, and easy-to-teach procedure. Surgery 1995; 118: 879-83.

46. UGARTE H. Experiencia de traqueostomía percutánea en una unidad de cuidados intensivos regional. Rev Chil Med Intensiva 2002; 17: A78.

47. Kost KM. Endoscopic percutaneous dilatational tracheostomy: a prospective evaluation of 500 consecutive cases. Laryngoscope 2007; 115: 1-30.

48. Paran H, Butnaru G, Hass I, Afanasyv A, Gutman M. Evaluation of a modified percutaneous tracheostomy technique without bronchoscopic guidance. Chest 2004; 126: 868-71.

49. Ben Nun A, Altman E, Best LA. Extended indications for percutaneous tracheostomy. Ann Thorac Surg 2005; 80: 1276-9.
50. Biankenship DR, Kulbersh BD, Gourin CG, Blanchard AR, TERRIS DJ. High-risk tracheostomy: exploring the limits of the percutaneous tracheostomy. Laryngoscope 2005; 115: 987-9.

51. Heyrosa MG, Melniczek DM, Rovito P, Nicholas GG. Percutaneous tracheostomy: a safe procedure in the morbidly obese. J Am Coll Surg 2006; 202: 618-22.

52. Massick DD, Powell DM, Price PD, Changsl, Souires G, Forrest LA, Young DC. Quantification of the learning curve for percutaneous dilatational tracheostomy. Laryngoscope 2000; 110: 222-8.

53. Romero C, Cornejo R, Gálvez R, Lanos O, Larrondo J, SCHONSTEDT V ET AL Traqueostomía percutánea: curva de aprendizaje. Rev Chil Med Intensiva 2006; 21: A185.

54. Friedman Y, Fildes J, Mizock B, Samuel J, Patel S, ApPaVu S, RoberTs R. Comparison of percutaneous and surgical tracheostomies. Chest 1996; 110: 480-5.

55. Holdgaard HO, Pedersen J, Jensen RH, Outzen KE, Midtgaard T, Johansen LV ET AL. Percutaneous dilatational tracheostomy versus conventional surgical trachesotomy. A clinical randomized study. Acta Anaesthesiol Scand 1998; 42: 545-50.

56. Porter JM, Ivatury RR. Preferred route of tracheostomy-percutaneous versus open at the bedside: a randomized, prospective study in the surgical intensive care unit. Am Surg 1999; 65: 142-6.

57. Freeman BD, Isabela K, Cobb JP, Boyle WA $3^{\mathrm{RD}}$, SCHMieg RE JR, KoLef MH ET AL. A prospective, randomized study comparing percutaneous with surgical tracheostomy in critically ill patients. Crit Care Med 2001; 29: 926-30.

58. Blankenship DR, Gourin CG, Davis WB, Blanchard AR, Segbt MW, TerRis DJ. Percutaneous tracheostomy: don't beat them, join them. Laryngoscope 2004; 114 : 1517-21.

59. Romero C, Larrondo J, Le-Feuvre O, Gálvez R, Ruiz M, MENDOZA Let AL Traqueostomía percutánea: tiempo para su ejecución. Rev Chil Med Intensiva 2005; 20: A153.

60. Baccheta MD, Girardi LN, Southarde J, MackCa, Ko W, TorTolani AJ ET AL. Comparison of open versus bedside percutaneous dilatational tracheostomy in the cardiothoracic surgical patient: outcomes and financial analysis. Ann Thorac Surg 2005; 79: 187985.

61. Kollef MH, Von Harz B, Prentice D, Shapiro SD, Silver P, St John R, Troviшon E. Patient transport from intensive care increases the risk of developing ventilator-associated pneumonia. Chest 1997; 112: 765-73.

62. Beckmann U, Giwes DM, Berenholtz SM, Wu AW, Pronovost P. Incidents relating to the intra-hospital transfer of the critically ill patients. An analysis of the reports submitted to the Australian Incident Monitoring Study in Intensive Care. Intensive Care Med 2004; 30: 1579-85. 